\title{
La juventud y sus límites operativos
}

\author{
ANTONIO ALAMINOS \\ INSTITUTO DE DESARROLLO SOCIAL Y PAZ \\ UNIVERSIDAD DE ALICANTE
}

\section{Resumen}

Los estudios de juventud representan una labor muy significativa en la investigación en Ciencias Sociales. Tanto en esfuerzos presupuestarios como académicos. No obstante, la revisión de la mayoría de estos estudios refleja una notable inconsistencia conceptual. Esta fragilidad conceptual permite un empleo ideológico, donde la elaboración de científica de categorías se sustituye por los prejuicios culturales del científico

Palabras Clave: Juventud, concepto, metodología, retórica, emancipación

\begin{abstract}
Research about young amount an important share of the Social Science studies. Nevertheless, the review of the majority of these studies reflects flimsy concepts. This conceptual fragility permits the ideological use, replacing the elaboration of the scientist categories by the cultural prejudices of the scientist.
\end{abstract}

Keywords: Youth, methodology, rhetorical, emancipation 
Con frecuencia los conceptos teóricos que los investigadores empleamos para analizar la sociedad se comportan como la botella de la metáfora de Wittgenstein. Cuanto más transparentes parecen, más impenetrables. Envuelven y encapsulan los fenómenos sociales de forma que fragmentan y distorsionan la realidad social. El concepto de juventud corresponde a esta categoría embotellada, y entre otros intentos, este escrito intenta explorar en búsqueda de la salida que permita derramar y reintegrar este segmento a la sociedad. En primer lugar, deberemos preguntarnos si Juventud es una categoría social o un concepto sociológico. La diferencia aparece ligada a las perspectivas etic y emic. En otras palabras, juventud es un concepto de empleo interno a la sociedad, que adquiere un significado por consenso, histórico e ideológico (realidad definida como tal y por ello con consecuencias sobre los comportamientos) y es un concepto externo, con capacidad heurística y predictiva, más allá de la realidad ideológica que le conceden los individuos. Es otra palabras, es un tipo social (Etiquetamiento) y es una tipología sociológica (Weber)

Lo cierto es que la categoría social de joven se superpone sobre una realidad concreta, la incorporación como fuerza de trabajo al mercado. En ese sentido, supone un rompeolas donde apreciar las tendencias estructurales de la economía en su redefinición de las relaciones laborales. La incorporación al mercado implicaría emancipación y articulación de una nueva unidad familiar con relación independiente con el Estado. En ese sentido, las edades articulan derechos y obligaciones que conviven a espaldas de la interpretación social. El mismo Estado considera diferentes edades limite. Aún cuando el cambio de tendencia estructural afecta a todos los segmentos sociales, es en la incorporación donde se nota con mayor eficacia. La precarización de la sociedad en su conjunto se inicia por el principio, en la incorporación al mercado de trabajo. Con el tiempo, tal y como esta sucediendo, el envejecimiento permitirá que los trabajadores solo conozcan esta situación precaria en su relación con el capital. En definitiva, asistimos a una reestructuración profunda, durante muchos años invisible (la cuestión era el retraso en la emancipación concertándose en la salida, familia, y no en la llegada, mercado) pero de prolongado efecto. Esta tendencia profunda de reestructuración del mercado de trabajo tiene un efecto de reacomodo ideológico en las clases medias. Reacomodo consecuencia del miedo al descenso de clase de sus hijos.

\section{La botella teórica}

Juventud ¿es un recipiente donde se recogen elementos coherentes en sus características definitorias o juventud es una "botella" que encierra una diversidad social?. Las tipologías, tanto expresas como referidas a categorías sociológicas, suponen un potencial importante entre las herramientas analíticas del sociólogo. No obstante, en la medida que el procedimiento hunde sus raíces en el "humus" cultural de cada sociedad, es importante en su empleo explicitar el procedimiento seguido para su construcción reduciendo al mínimo la retórica argumental utilizada para hacer convincente el tipo o categoría construida.

En primer lugar, es imprescindible el deslinde entre la generación social "espontánea" (en el sentido de no evaluado) y su empleo como herramienta de 
interpretación y explicación sociológica. Es evidente que estas tipologías producidas socialmente definen un objeto de estudio con interés por sí mismas, tanto en su vertiente cultural (arquetipos y mitos) como cotidiana (donde la tipologización actúa catalogando y prescribiendo expectativas de comportamiento). En ese sentido, el procedimiento social "espontáneo" de categorización es susceptible de ser interpretado en clave funcionalista o de conflictos según el papel que se le atribuya. La tipologización es "per se" un mecanismo social cuya realización colectiva importa al sociólogo. Constituye por ello simultáneamente un objeto de investigación y una heurística tanto de la investigación como de la teorización social. Esta utilidad metodológica alcanza la actividad misma tanto de definir actores (segmentación, tipologías, conjuntos de rol-estatus, etc.) mediante extracción cuantitativa como cualitativa.

En tanto que objeto de estudio los procesos de tipologización social son susceptibles de lecturas diversas. En la tradición funcionalista destaca Klapp, ofreciendo dos versiones. La primera de ellas reconoce las tipologías con un componente "cultural" legendario, elaboradas desde la "conciencia colectiva" y que cumplen una función de "mito" o arquetipo. De este modo, para Klapp (1949) un tipo social se podría definir inicialmente como un concepto colectivo de persona o conducta que aparece frecuentemente en el folklore o la literatura. En esa definición, se incorporaría un repertorio importante de funciones ligadas a la ejemplaridad o control que determinados "tipos" pueden desarrollar. En ese sentido, existe una dimensión cultural de los tipos que debe considerarse, en la medida que interfiere sobre la elaboración o desarrollo de "tipificaciones" con intencionalidad científica. Un ejemplo de ello es el continuo cruce conceptual en los trabajos de Freud con las mitologías griegas, donde con frecuencia el ejemplo reemplaza a la explicación. En una segunda dimensión definitoria aparecen los tipos sociales con un grado menor de codificación y "reificación", ejerciendo una función mucho más operativa en la vida cotidiana. De este modo, Klapp (1958) ofrece una segunda definición donde el "tipo social" constituye un conjunto difuso de roles, no codificado o racionalizado, que actúa ayudando a los individuos a orientarse en el sistema social. Constituiría, en definitiva, una especie de mapa latente de la estructura de roles existente en un momento concreto. Sin embargo, para Klapp este mapa no constituye una herramienta desarrollada por el científico para elaborar categorías sociales, sino más bien un método de etiquetado utilizado socialmente de forma espontánea. Así, para Klapp, la definición de tipo social se encuentra muy próxima a la noción de "imágenes de status" propuesta por Goffman (1959). Los tipos sociales suponen un doble objeto de investigación, ya elaborados espontáneamente desde la conciencia colectiva o como método de identificación de status. Por ello, los tipos sociales producto de una actividad espontánea de etiquetado desarrollarían una "función social" reconocible y analizable. Este carácter funcionalista es más explícito en otros autores como Booth y Blair, aunque para ellos los tipos sociales no constituyen tanto un mapa de una estructura social "institucionalizada", sino más bien una forma de etiquetar aquellos conjuntos de roles no convencionales que van surgiendo en un sistema sociocultural ("sin techo", "yuppies", "posmodernos", etc.); con ello se concentran en las categorías emergentes que son reconocidas y empleadas públicamente. En ese sentido, no constituye una definición nueva de tipo social, 
sino que más bien desplazan el acento desde los tipos institucionalizados a los emergentes. No obstante, la función de etiquetado social se mantiene en los mismos términos de reconocimiento e identificación para el conjunto de la sociedad.

Desde un punto de vista menos global, son varios los autores que han destacado la función de reconocimiento e identificación que las tipologías ejercen en la dinámica de pertenencia a grupos. Así, por ejemplo, Strong $(1943,1946)$ o Taub y Leger (1984). Para Strong, un "tipo social" es un constructo elaborado por un grupo mediante la selección y abstracción de formas de conducta específicas desarrolladas por algunos de los miembros del grupo. El criterio de selección de las conductas es simplemente el interés para el grupo. Esta interacción entre actitudes grupales e individuales que existe detrás de un tipo social determinaría los roles que ejerce un individuo en su entorno social próximo.

Destaca en definitiva en estos autores la consideración de los tipos sociales como categorías generadas espontáneamente, ya sea por la sociedad en conjunto o bien dentro de los grupos en su proceso de definición, que ejercen una función social de regulación y catalogación de pautas de comportamiento esperadas. No obstante, aún manteniendo que los tipos constituyen catalogaciones sociales, estas pueden responder a un reflejo de los procesos de exclusión y conflicto dentro de la sociedad. En dicho planteamiento destacan Glick y Kinloch. Para ellos, la elaboración de tipos sociales no constituye un proceso espontáneo de categorización social, sino que por el contrario responde al etiquetado propio de los estereotipos, destinado a la exclusión de grupos, clases o razas. Así, para Glick (1955) los tipos sociales son construcciones sociales generadas por los grupos, mediante las que se etiqueta con una finalidad excluyente a determinadas personas con un modo específico de comportamiento.

A diferencia de los autores anteriores, donde las tipologizaciones son tomadas como objeto de estudio, en la medida que se generan en la vida cotidiana, otros investigadores han considerado la construcción de tipos como una herramienta útil en el análisis de la realidad social. Así, para Reading (1977) un tipo social es aquel elaborado desde fuera de la sociedad y no desde dentro de ella. El tipo social sería, por lo tanto, una descripción resumida y objetiva de las características que posee una categoría concreta de personas. Esta aproximación por la que se elaboran tipos sociales para formar una tipología sociológica es sostenida también por Becker (1976) y otros autores.

Entre los antecedentes ilustres de la sociología en el empleo y proposición de las tipologías como herramienta de conocimiento destacan M. Weber y G. Simmel. Ambos autores señalaron la importancia del empleo de tipos sociales para mejorar el conocimiento y comprensión de la sociedad. En el caso de Simmel, los tipos sociales aparecen como una composición de determinadas características psicológicas, personalidad, temperamento o mentalidad formada por condiciones estructurales, o ecológicas. El planteamiento sería desarrollado por Park (1928) al describir al "hombre marginal" como un tipo concreto de personalidad y con una forma característica de comportamiento. No obstante, postular la coincidencia entre tipo social y personalidad ha sido objeto de oposición para algunos autores. Burgess 
(1968), plantea que el término "tipo social" no se refiere a mecanismos de la personalidad sino a las actitudes y valores que se derivan como copias de las que ofrece la sociedad. De esta forma, un tipo social aparece como consecuencia del papel asumido por una persona y que le ha sido asignado por la sociedad. La argumentación de Burgess en contra del peso que pueda adquirir la personalidad para definir un tipo social se apoya en que la personalidad se adquiere en la infancia y adolescencia, y que por lo tanto sólo podrían ser adoptados en ese mismo período. Así, los cambios en los tipos sociales no plantean cambios en la personalidad básica. Este aspecto sería destacado por Zorbaugh (1968) al definir el tipo social como una constelación de actitudes que forman una pauta de personalidad, no heredada sino desarrollada en una situación social concreta. Coser (1974) describe los tipos sociales como aquellas características destacables de los individuos que tienen una posición notoria en la sociedad y están motivados por lo tanto a desarrollar un tipo especial de comportamiento de rol. En ese sentido el planteamiento de Coser es bastante vago, tanto por carecer de una definición precisa (por ejemplo, posición notoria), como porque en él aparece el empleo híbrido del tipo social, donde se cruzan los rasgos socialmente definidos con la caracterización del sociólogo que los elabora.

Precisamente la doble naturaleza de la tipificación (como objeto, como método), sitúa la actividad del sociólogo sobre un cruce de caminos, donde las herramientas de investigación son tomadas (vienen dadas) bajo su forma de producto cultural o social. Sin embargo, es más que evidente que el proceso de estereotipia puede contaminar de prejuicios no comprobables empíricamente estas tipologías. Valga como ejemplo en este caso la tipología "juventud", a la que con frecuencia se atribuyen cualidades psicológicas y de carácter (rebeldía, conflicto, no conformismo, etc) que son dependientes del contexto histórico, de la clase social, del entorno familiar, etcétera y no predicables intrínsecamente de un tipo genérico denominado "juventud". En ese sentido, si los jóvenes frustran expectativas de comportamiento puede ser simplemente por que el "tipo" responde a estereotipos ahistóricos.

\section{Sobre los jóvenes como tipología}

Con frecuencia los investigadores sociales empleamos los grupos de edad. Estos pueden aparecer directamente operativizados en tramos de edad o indirectamente etiquetados en categorías como infancia, adolescencia, juventud, etc. En ese sentido, se han desarrollado diferentes revisiones tipológicas como las de Forner (1980) o más recientemente Bettin (1999). La cuestión fundamental es si los grupos de edad son una mera construcción estadística, que permite operativizar de forma limpia e inmediata (por lo tanto definiendo meras categorías numéricas), o realmente pretende expresar y recoger tipos con diferencias significativas y existencia real en la sociedad de acuerdo a los planteamientos de autores como Bengtson, Furlong y Laufer, (1974) o Elder1975). Porque incluso en el caso en que el investigador no indique (como es habitual) los rasgos diferenciales en los grupos de edad, el lector tenderá (entimema) a reconocer diferencias entre ellos en función a su experiencia sobre los ciclos de vida, condiciones económicas, sociales o culturales. Ciertamente no es un asunto que concierna exclusivamente a la técnica, por el contrario su interés teórico es sustantivo. En la medida que la construcción de 
grupos de edad refieren a tipologías (de modo explícito o implícito) y estas tipologías son construcciones sociales históricas empleadas en la vida cotidiana, (por ejemplo en relación a la construcción misma de la identidad Fogarty, 1973) se evidencia la necesidad de tomar dichas tipologías primero como objeto de estudio (elementos que intervienen en su construcción social y cultural, cambios experimentados con las trasformaciones sociales y económicas, etc.) y segundo, desarrollar su empleo analítico como construcción epistémica (Bourdieu, 1984) que ayuda a explicar la realidad social y puede o no concordar con su definición social cotidiana. Simplemente, cerrar la brecha entimemática que permite penetrar el lenguaje cotidiano acrítico (con todo lo que eso implica) en el metalenguaje del investigador.

De hecho las referencias numéricas en la edad que pueda corresponder a dichos tipos se modifican con las trasformaciones sociales, tal y como mostró Nissel (1982) para el caso Británico. En definitiva, dado que los grupos de edad parecen no ser (ni pretenderlo) exclusivamente un etiquetado numérico de la realidad, se impone considerar la variabilidad interna que contiene, para en el caso que sea pertinente, "desguazar" una tipología amplia y reductora como es la de juventud y desplegarla (si procede) en tipos coherentes definidos sustantivamente. Quizás entonces debiera, restringirse la etiqueta de jóvenes no a un tramo de edad, sino a un grupo específico con características concretas que debieran, sin duda, ser explícitas, simplemente para poder ser discutidas. En esta rápida evaluación del concepto juventud en tanto que tipología social, vamos a considerar algunos de los criterios principales que para algunos autores (Almog, 1998) contribuyen a la conformación de tipos sociales, como son los de carácter psicológico y ocupacional.

En ese sentido, la edad y su empleo como indicador privilegiado en la operativización de la juventud aparece condensando una importante polisemia. Ciclo, generación, maduración, período, la edad es un "indicador" genérico (al igual que un "comodín" en los juegos de cartas) del que debemos especificar su significado si realmente se pretende utilizar para comprender el mundo en que vivimos. Así, por ejemplo, la edad puede aparecer en una explicación expresando tanto envejecimiento (maduración psicológica), como posición en un "ciclo vital", como "muesca" que expresa el impacto de un período (una transición política, por ejemplo) o incluso como "actor social" con entidad propia (concepto de "generación" desde una perspectiva histórica), y con capacidad explicativa de las trasformaciones sociales y políticas.

En general, plantear la juventud como una etapa psicológica, dentro de un proceso global de maduración, o formación de carácter posee profundas raíces culturales, tal y como refleja la clasificación que ofrece Shakespeare (inspirada en Hipócrates) al resumir la vida del hombre en siete etapas, diferenciadas por las trasformaciones de temperamento. Con ello, el concepto (no operativizado en la sociología) de maduración se cruza sobre otras tipologías psicológicas. La relación entre edad y maduración se evidencia en la dimensión legal. Quizás donde más explícitamente se aprecie es en el Derecho canónigo, dado que la edad mínima para contraer matrimonio desaparece como límite al constar que "ó antes si la malicia suple a la edad". En definitiva la edad es un indicador de madurez, inválido cuando la 
malicia ya está presente. Desde esta perspectiva, se hace evidente que la madurez es un fenómeno que no acontece de modo sincrónico y en un momento concreto. Por ello, dentro del criterio general necesario de la edad, se particulariza y excluye aquellas tipologías (malicia) que no cumplen. En resumen, la edad es indiciadora de una propiedad, cuando esa propiedad no se cumple más (ya no existe) la edad no importa. ¿Es la edad lo que nos importa para definir una tipología de "juventud” o aquello que indicia?

Si la "malicia", entre otros aspectos, contribuye a la borrosidad de entrada en la categoría "joven" desde el punto de vista de la maduración, la ocupación aparece especialmente ligada a la salida de ésta. Existe un cierto "conglomerado" conceptual que asocia los conceptos de emancipación, ocupación y juventud, entre otros. No obstante, juventud y emancipación no son sinónimos teóricamente, si bien tienden a ejercer de ese modo en la práctica. Existe un grupo importante de jóvenes emancipados (en precario o no) antes de los 20 años. Si atendemos a lo que se considera socialmente deseable, puede apreciarse con claridad que la edad adecuada para independizarse se encuentra entre los 24 a 25 años ${ }^{1}$. Esta opinión se corrobora con aquellos que se emanciparon antes de esa edad, en la medida que tienden a pensar que fue demasiado pronto. La mayor parte de los entrevistados no emancipados menores de 30 años y mayores de 25 piensan que se emanciparan más tarde de lo que es deseable. En ese sentido, emanciparse entendido como vivir independiente de la unidad familiar de origen, tiene un límite relativamente claro en los 25 años en el imaginario de lo socialmente deseable. Son varias las razones para no emanciparse, si bien la más extendida es la carencia de recursos económicos. De este modo, la ocupación en trabajos precarios no permiten la emancipación con unas ciertas garantías en el estilo de vida. El miedo de las clases medias (en un sentido amplio), al descenso socioeconómico de los hijos impone las condiciones familiares de convivencia para enfrentarse a la nueva situación laboral. Sin embargo, la emancipación es un concepto ligado a los "ciclos vitales" y no estrictamente al de juventud. En el caso que la no emancipación sea un rasgo definitorio de la juventud, surgirían problemas con un colectivo que se difumina de acuerdo al incremento de la edad. Sin duda, la emancipación tardía es en parte responsable del “estiramiento" operativo que arrastra al tipo "juventud" hasta los 30 o 32 años. No obstante, más que contribuir a la definición teórica de la categoría joven, este estiramiento histórico (dependiente en definitiva de la coyuntura económica) permite distinguir una cierta "distracción" sociológica. La categoría "joven" "enmascara" como situación temporal y de tránsito lo que se desarrolla de modo estructural y estable. Si un individuo de 30 años lleva 7 de ellos con trabajos temporales, discontinuos o directamente desempleado, puede llamársele joven o también adulto empezando a vivir las nuevas formas de organización del mercado de trabajo. El nombre, que duda cabe, importa.

Parecería necesaria una mayor reflexión y debate sobre el concepto sociológico de juventud, su significado teórico, así como los indicadores asociados a su operativización como categoría genérica, en el caso que esto fuese deseable. De otro modo, el análisis y la interpretación se convierten sistemáticamente en

\footnotetext{
${ }^{1}$ Datos: Estudio CIS 2262 “Juventud y entorno familiar”. Octubre 1997.
} 
exploratorias, al ser consecuencia de una definición borrosa en el origen (jóvenes de 15 a 30 años, por ejemplo) y no producto de una realidad definida teóricamente (donde por ello, los cambios puedan ser significativos y no meras anécdotas venidas de una definición operativa fungible).

Esta distorsión es claramente visible cuando se enfoca la cuestión desde el mercado de trabajo. Hidalgo Vega y Pérez Camarero². Destacan como "Ello, a su vez, repercute en una transformación de la percepción colectiva de la juventud, elevándose el listón de separación entre el grupo juvenil y los considerados mayores. No es difícil encontrar estudios sociológicos en los que la edad juvenil se hace llegar a los treinta y cinco años, circunstancia asociada no solo con la mejora de las condiciones de vida, sino también con la elevación del periodo educativo y el retraso en el acceso al mercado laboral para la mayoría de la población. Simultáneamente, encontramos múltiples trabajos demográficos y económicos relativos a países en desarrollo en los que se considera juventud trabajadora al tramo de edad entre 14 a 24 años, segmento reconocido y utilizado por Naciones Unidas para delimitar la edad juvenil. Reconociendo el relativismo cultural, económico e histórico de la etapa denominada juventud, hemos centrado este trabajo sobre la franja de edad que abarca desde los 16 años, edad laboral mínima en España, hasta los 29, ambos años incluidos. En todo caso, cualquier listón separador de dos fases en la evolución del individuo ha de entenderse como un acuerdo meramente convencional que permite establecer unos parámetros más o menos fijos de medida y análisis". Joven es un concepto cultural diverso y las consecuencias de este hecho se reflejan inmediatamente en la multiplicidad de sus definiciones operativas. Ciertamente no es un hecho novedoso el que la clasificación de las edades del hombre ha sido una cuestión compleja. Al fin y al cabo, tan operativa podríamos considerar la división en siete etapas que hace Shakespeare (varias de las cuales podrían ser consideradas como jóvenes) apoyándose sobre las diferencias de temperamento, como la clasificación enigmática de la esfinge edípica, que nos permite llamar a los ancianos tercera edad. En las sociedades modernas, sin "ritos sociales de paso" bien establecidos, el investigador tiende a encontrarse en una situación de tal familiaridad con el concepto que le hace trasparente a su problematización explícita; y sin embargo sabemos que el contenido (referente empírico del concepto) se esta trasformado. "Joven" es un concepto que reúne suficiente cotidianidad al colectivo de investigadores como para que desde el análisis de las ciencias sociales devenga casi obvio. La consecuencia inmediata es que en muchos estudios de ámbito tanto nacional como internacional los contenidos operativos del concepto "juventud" se dan por conocidos. Esta es una situación que se produce en muchos otros campos de la investigación social, donde la retórica substituye a la ciencia (R. Edmondson, 1984, Perelman 1979). Básicamente, en el tratamiento del concepto juventud podemos considerar dos estrategias complementarias, apoyadas ambas sobre la idea de grupo de edad: el efecto retórico epitémico y el entimémico.

La estrategia "entimémica” permite al investigador aplicar la ambigüedad de toda etiqueta convencional, obviando precisamente la necesidad de una definición operativa donde se expliciten los limites (conceptuales o numéricos) del grupo de

\footnotetext{
${ }^{2}$ Hidalgo Vega y Pérez Camarero Aspectos salariales de los jóvenes trabajadores INJUVE. Madrid. Pg. 13-14
} 
edad “joven”, así como las razones que justificarían dicha agrupación de individuos. Recordemos que toda distinción requiere de una diferencia, y es precisamente la explicitación de las diferencias del grupo de edad joven frente a otros grupos lo que se echa en falta en muchas investigaciones. Una opción generalizada de validación consiste en generar una agrupación de individuos y someterla posteriormente a contraste con otros grupos para caracterizar las diferencias que le definen operativamente. Este procedimiento peca obviamente de ser una falacia desde un punto de vista metodológico, en la medida que las descripciones que pudieran encontrarse posteriormente no justifican los criterios de acotación conceptual que explicarían la formación inicial de un grupo de edad.

Debe reconocerse no obstante, que todo limite a un grupo de individuos padece necesariamente de borrosidad. Esto es especialmente cierto si aplicamos la percepción social tradicional donde los jóvenes son seres en transito y cuya ciudadanía plena esta en cuestión; baste para ello recordar la celebre afirmación "la juventud es una enfermedad que se cura con la edad". Sin embargo, y como ejemplo, la noción de joven como etapa de maduración e incorporación a la sociedad se encuentra traspasada por múltiples contradicciones. Considerar al joven como etapa de transito debe explicitar hacia que fractal social (grupos) o psicológico (personalidad): maduración emocional, intelectual, política, económica, sexual, creación de una nueva unidad familiar, etc. La complejidad de las sociedades actuales asincroniza la incorporación de los jóvenes a la sociedad de tal forma que deviene en una pertenencia y no pertenencia simultanea a diferentes grupos sociales. Así, un joven podría ser adulto político, joven económico, adulto intelectual, joven emocional y así un largo etcétera. En todo caso, la aplicación de una definición conceptual al grupo de edad "joven" necesita que esta se haga explícita en un correlato medible bajo la forma de un conjunto de indicadores o variables que expresen las diferencias entre grupos de edad y que consecuentemente peculiariza a los jóvenes.

Una segunda estrategia, “epitémica”, enfrenta la cuestión de acotar operativamente la noción del grupo de edad “joven" mediante la simple determinación de unos tramos de edad. Aquí también son abundantes los ejemplos en la investigación internacional (Kaufman y Spilerman 1982), donde la construcción de un intervalo de edad etiquetado como joven permite obviar una información tan importante como es el definir las diferencias entre aquellos agrupados dentro de la categoría "joven” y aquellos otros que por su edad están próximos a entrar en ella o bien han dejado de pertenecer. Dar por evidentes las relaciones entre los grupos de edad que se atribuyen la operativización de los jóvenes y el contenido del concepto es un sobreentendido quizás excesivo. Especialmente por las condiciones históricas y multiculturales del fenómeno. Podemos apreciar un ejemplo de esto anterior desde la óptica del caso español.

Precisamente la prolongación de la edad a 29 años viene motivada por su asociación al concepto (diferente) de emancipación. De este modo, se les considerara como jóvenes mientras su probabilidad de emancipación (empleo estable y vivienda independiente del domicilio familiar) es baja. Esta definición de joven sobre la base de la emancipación lleva a evidentes paradojas y a una 
ampliación del tramo de edad que en algunos casos llega más allá de los 30 años. Esta situación ambigua en la edad de cierre (24 o 29 años) es claramente producto de la borrosidad del concepto de responsabilidad social plena. A. Cavalli (1997) define para el caso italiano dos conceptos de joven, directamente ejemplares de la variabilidad en la definición de muestra que encontrábamos para España. La “juventud corta”, (entre 15 y 24 años) y la "juventud larga” (entre 24 y 29 años). Los márgenes de la definición vienen facilitados por el abandono de la adolescencia (criterio sexual) y la noción de "responsabilidad civil". Dentro de la mencionada división operativa, un aspecto importante proviene de la relación que A. Cavalli postula entre tipos de juventud y clase social. La prolongación de la juventud seria un epifenomeno de clase. Ciertamente, es difícil determinar el elemento de corte entre "joven" y "adulto", si bien refinamientos conceptuales como el mencionado ayuda, sin lugar a dudas, a profundizar en las características de dicha segmentación.

Para el caso español, la confusión entre el concepto operativo “joven” y el de "emancipación" conduce a consecuencias interpretativas interesantes; así, producto de la confusión implícita de los términos juventud y emancipación, se encuentran las interpretaciones de las relaciones entre padres e hijos bajo la luz distorsionada de un concepto erróneamente definido. Por ejemplo, podría decirse de un padre que es tolerante con su hijo de 15 años admitiéndole una gran autonomía. Sin embargo, ¿podría decirse lo mismo si el hijo tiene 29 años, aun cuando no este emancipado y por ello se le denomine equívocamente “joven”?. Como podemos apreciar, la definición correcta del concepto joven no es trivial, dado que determinara interpretaciones de la sociedad en múltiples dimensiones: relación familiar, emancipación, valores, actitudes, etc.

Hablar de jóvenes es hablar de familia. El permanecer en el domicilio de los padres es un fenómeno sobre todo de las sociedades del sur de Europa. Esto podría tener relación con las teorías de E. Todd o con la precariedad del Estado ¿cuáles son estos valores que importan a los jóvenes en porcentajes entorno al 90\%?. La familia, la amistad, el trabajo, el tiempo libre y ocio, ganar dinero, la salud y la vivienda. No existe conflicto familiar en términos de conservación de status, dado que lo que preocupa y aprecian los jóvenes es aquello que comparten en objetivos con los padres. De hecho, la felicidad en la pareja, es decir, una emancipación exitosa depende para los "jóvenes" de la economía, tener unos ingresos adecuados (90\%), y una buena vivienda (70\%). En lo personal se pide fidelidad (98\%) y relaciones sexuales satisfactorias (92\%). Dada la importancia que se concede a la comprensión y tolerancia $(96 \%)$ de la pareja, pasan un plano secundario las ideas políticas, religiosas y otras diferencias "ideológicas". La jerarquía de valores para alcanzar la felicidad en la emancipación es coherente y racional. Con ingresos adecuados ya no importan las diferencias de clase, fidelidad y relaciones sexuales satisfactorias conceden la estabilidad emocional así como la tolerancia y comprensión evitan las discrepancias y disputas.

Una definición elástica de joven (definida como emancipación) permite análisis donde padres permisivos "aguantan" a los hijos. La realidad es una tensión familiar importante orientada a la conservación del status. La permanencia de los hijos en casa es parte de una estrategia económica de la clase media. De este modo, 
la felicidad depende en un grado importante de la vivienda, el trabajo fijo, el dinero. Por ello la emancipación económica se produce retrasada con respecto a otras, como son la sexual. Esta estrategia es de clase, como apuntaba Cavalli. De este modo, la emancipación en la juventud corta, antes de los 24 , se asocia a una emancipación temprana y posiblemente traumática. La juventud larga es una estrategia de clase media y en ese sentido un artefacto histórico que debería ser considerado con cuidado en el análisis sociológico.

Cuando se considera a los jóvenes de la actualidad, uno de los aspectos que más se acostumbra a destacar es el referido a la prolongación de la permanencia de estos en el hogar familiar. Ciertamente si consideramos la situación actual desde la perspectiva de lo sucedido con generaciones anteriores, la tendencia que encontramos es ciertamente diferente. Durante mucho tiempo, la idea de joven ha sido relacionada con la noción de conflicto e independencia, especialmente con relación a los padres. En ese sentido, los conceptos de joven y rebeldía tienden a estar asociados en la mente de todos. Sin embargo, el contexto social y económico en el que se desenvuelven los jóvenes hoy en día es bastante ajeno a los conceptos mencionados.

La noción clave para comprender las actitudes, comportamientos y opiniones de los jóvenes es "integración”. Los jóvenes generalmente desean participar de forma activa en una sociedad que en términos globales les atrae. Así, apreciamos como en la mayoría de los jóvenes, tiene una posición central la noción de emancipación estable. Hablar de jóvenes es hablar de un grupo social que manifiesta un deseo explícito de participar con normalidad en la vida cotidiana. Esto implica, evidentemente, la creación de una unidad familiar propia. El perfil de joven basado en los conceptos de independencia, rebeldía, afirmación frente a los padres o la autoridad, no tiene nada que ver con el perfil medio de los jóvenes actuales.

Resulta evidente que si las expectativas de los jóvenes ha cambiado, su relación con los padres también se ha modificado. Ciertamente el tópico de padres e hijos en conflicto ha sido reemplazado por una convivencia más o menos pacifica. No podía ser en buena lógica de otro modo, dado que los deseos de integración social que manifiesta explícitamente los jóvenes encuentran un eco favorable inmediato en sus partes. Por tanto, si el joven rebelde se encontraba en una situación permanente de enfrentamiento con sus padres, el joven cuyo deseo es formar parte de la sociedad, mediante un trabajo estable, una vivienda, etcétera, encuentra una disposición mucho más comprensiva en su entorno familiar.

Encontramos, por tanto, que el cambio de actitud en las generaciones de jóvenes actuales, implica un cambio simultáneamente en el entorno de las relaciones familiares. Allí donde se encontraba conflicto aparece consenso, como expresión directa del acuerdo implícito o explícito entre el proyecto de vida del joven y el deseado por sus, padres. Este cambio de clima, donde se encuentran una expresión mayor de solidaridad familiar, hace decir a muchos analistas que la permanencia en el hogar paterno de muchos jóvenes, viene propiciado por una mayor tolerancia por parte de sus progenitores. Esto supone una verdad incompleta. La permanencia prolongada de los jóvenes en el domicilio de sus padres viene sobre todo 
propiciadas por el hecho de compartir objetivos vitales. Los hijos desean algo que sus padres desean para ellos, las dificultades actuales para conseguirlo es una de las razones que propicia esa tolerancia por parte de los padres. No existen conflictos básicos de intereses. Generaciones anteriores, planteando objetivos vitales diferentes aquellos deseados por sus padres para ellos, generaban un contexto de conflicto que en frecuentemente se resolvía en forma de emancipación del joven. Como venimos mencionando, no es el caso en la actualidad. Generalmente no existe situación de conflicto y por lo tanto el clima familiar es más solidario en la consecución de unos objetivos.

No es solamente el clima familiar diferente que encontramos en la actualidad lo que produce unos padres más tolerantes (desarrollado sobre el consenso con respecto a los objetivos vitales de los hijos) sino también el hecho de que no basta con la prolongación de la permanencia de los hijos en casa de sus padres para que el concepto de joven retenga todos sus significados. Así, un hijo puede vivir en casa de sus padres por no encontrar un empleo estable, pero no por ello podemos atribuirle todas las características de un joven como tal. Los esquemas culturales permanecen, de tal forma que un "joven" de 28 años no puede ser tratado por sus padres como un joven de 18. Los padres son conscientes en mayor o menor grado, de que su hijo esta más próximo a ser considerado socialmente como un hombre y no como joven inmaduro. La noción de joven implica no solamente una etapa de transición social, como paso previo en sentido amplio a la formación de una nueva unidad familiar, sino que asimismo asocian la idea de una cierta inmadurez. Ciertamente, podemos considerar como joven a una persona que depende económicamente de sus padres y vive en el domicilio de éstos. No obstante, la elasticidad teórica del concepto no encuentra un reflejo inmediato en la sociedad. Denominar joven a una persona de treinta años, sobre la base de las circunstancias en que vive (no emancipado) es factible en términos de definición operativa, sin embargo es bastante probable que su familia haya percibido cambios de comportamiento y actitud que le aproximan más a la edad adulta. Pensamos que tiene sentido adjetivar como tolerante a un padre que otorga una libertad amplía a su hijo de catorce años, sin embargo no es tan adecuado etiquetar a un padre de la misma manera cuando su hijo ya tiene más de veinticuatro (por ejemplo).

En resumen, los jóvenes actuales no aspiran a transformar la sociedad, sino que por el contrario una de sus máximas aspiraciones es poder participar de ella. Esto implica, que la emancipación no supone una estrategia de distanciamiento de la familia (crisis de enfrentamiento e identidad) y por lo tanto indirectamente de crisis de la cohesión social, si no que por el contrario supone netamente una estrategia orientada a la constitución de una nueva unidad familiar. En ese sentido, la emancipación de los jóvenes no se plantea como una discontinuidad con respecto a la familia de origen, si no que por el contrario, son esencialmente una aspiración de continuidad, y por lo tanto de integración social. Por ello la emancipación de los jóvenes es más un proyecto familiar que individual. La familia como vínculo de integración y de relación social ha adquirido un papel fundamental en la sociedad actual. 
Hemos considerado como elemento central en la relación del joven con su familia el hecho de compartir, en gran parte, los objetivos vitales del hijo. Estos objetivos vitales están orientados a obtener una emancipación de tipo estable. Debemos evaluar, por lo tanto, los rasgos de una emancipación estable en la cultura actual de los españoles. Así, en definitiva, hablar de una emancipación estable del joven es referirse a los conceptos de empleo estable y vivienda en propiedad. Desde el punto de vista de la sociedad española en general, lo óptimo para facilitar la emancipación de los hijos pasa por la obtención por parte de estos de un empleo estable. Un segundo objetivo vital para los hijos es el referido al acceso a corto o medio plazo a una vivienda en propiedad. Son precisamente las condiciones del mercado de trabajo, donde se generan básicamente empleos temporales, las que provocan que los jóvenes (y en ello reciben el apoyo de sus padres) no se emancipen cuando su principal fuente de ingresos proviene de un empleo temporal. Un objetivo prioritario para el joven, así como para su familia, es conseguir un empleo estable que le facilite ciertas garantías en su proyecto de emancipación y creación de una nueva familia.

Una situación semejante la encontramos cuando consideramos el problema de la vivienda. Para la sociedad española, lo ideal es la adquisición de la vivienda en propiedad. El alquiler es visto culturalmente como un mal menor o directamente como una etapa hacia la compra de una vivienda. En ese sentido, como hemos visto, existen una serie de estrategias orientadas a conseguir dicha finalidad (viviendas puente, acumulación de capital para adquisición de una vivienda, etcétera). Una de las características específicas del periodo que estamos viviendo consiste en que la emancipación de las jóvenes no es en absoluto un asunto exclusivo de éstos, sino que por el contrario, involucra a la unidad familiar de la que procede. En primer lugar destacamos el hecho de que ese retraso en la emancipación del joven no corresponde con una decisión particular del joven, si no que por el contrario se adecua en general a una estrategia familiar. La dimensión latente tras estas estrategias familiares refleja el deseo de conservación del estatus familiar. En ese sentido, una familia preferirá que su hijo se emancipe en condiciones de estabilidad y no de cualquier forma. Así, es lógico que los hijos permanezcan en el domicilio paterno mientras esperan unas condiciones favorables de emancipación. Precisamente, la importancia de la familia en la toma decisión por parte del joven para emanciparse es muy elevada. En grandes rasgos, las estrategias de conservación de status están apoyadas básicamente sobre las referidas a la de empleo para el joven, así como sobre las estrategias de adquisición de vivienda.

La familia ejerce un papel muy importante en las estrategias de empleo por parte del joven. Esto es apreciable directamente, al evaluar los procedimientos mediante los que aquellos que trabajan o han trabajado obtuvieron dicho empleo. La mayor parte de los jóvenes han obtenido un trabajo mediante contactos informales, fundamentalmente los desarrollados a través de la familia, ya sea porque un familiar trabaja allí o un amigo conocido por algún familiar. En ese sentido, la familia actúa solidariamente como agencias informales de búsqueda de empleo. Un apoyo solidario se detecta también en el acceso a la vivienda. 
La estrategia de adquisición de vivienda se apoya, fundamentalmente, sobre la facilidad que ofrece la familia al joven, tanto en la forma de apoyo económico para la adquisición de las viviendas como en otros casos facilitándole la vida familiar sin gastos, mientras acumula ahorros suficientes para la adquisición de una vivienda. Los padres, al igual que los hijos, consideran el alquiler de una vivienda como una situación precaria, no compatible excepto en condiciones excepcionales con la emancipación estable del joven. De hecho, esta disposición cultural hace que se genere el concepto de vivienda puente. Una vivienda puente es aquella de precio módico que se adquiere para no tener que pagar un alquiler, pero con la finalidad última de ser vendida para acceder a otra vivienda en propiedad más cercana a sus deseos. En ese sentido, la labor de acumulación de capital que efectúa el joven al ahorrar mientras vive con sus padres puede encontrar aplicaciones inmediatas en caso de ser necesario.

Como hemos podido apreciar, son varias las características que definen y peculiarizan a los jóvenes actuales, donde la emancipación se convierte generalmente en un proyecto familiar (en tanto que nueva unidad familiar que mantiene la integración social), tanto en origen como en destino. En este contexto, no cabe ninguna duda que las políticas públicas de apoyo al empleo estable, o en el peor de los casos, del empleo en general, así como con relación a facilitar el acceso a la vivienda por parte de los jóvenes, encuentran un importante eco social. No obstante, las tendencias de los mercados de trabajo contradicen las pretensiones de los jóvenes y sus familias, dado que la temporalidad y la movilidad laboral son predominantes. Dicha contradicción cultural es difícil de superar y sus efectos son directamente observables en la borrosidad de salida de la categoría joven. 


\section{Bibliografía}

Abramson P. (1976) "Generational Change and the Decline of Party Identification in America: 1952-1974" American Political Science Review, vol.70 n²

Alaminos A. (1987) Los jóvenes estudiantes Latinoamericanos en España. C.E.D.E.A.L. Madrid. Madrid.

Alaminos A. (1989) La transición ideológica en España. Informe CIS.

Alaminos A. (1990) Estudio sobre la Juventud Española. Informe C.I.S. Madrid.

Alaminos, A. (1994) "La cultura política de los jóvenes" en Martín Serrano, M. (dir) Historia de los cambios de mentalidad de los jóvenes entre 1960 y 1990. Madrid: Instituto de la Juventud

Alaminos A., Castejon y otros (1998) Los jóvenes alicantinos: vivienda, empleo y emancipación. Ayuntamiento de Alicante. Alicante.

Alaminos, A. Y Penalva, C. (1999) "Giovanni, socializzazione e ideología política in Spagna" en Bettin G. (dir) Giovanni e democracia in Europa. Vol II, Padova CEDAM. 1998

Almog, O.” The Problem of Social Type" Electronic Journal of Sociology

Allen, S. (1968) "Some theoretical problems in the study of youth", Sociological Review, Vol. 16, pp. 2319-31

Barroso Rival, C. Cuesta Ojeda, O. y Granados López, J. (1998) Libro blanco de la juventud en Canarias. (vol. I) ed. Dirección General de la Juventud.

Becker, S. H. (1976). "Art Worlds and Social Types." American Behavioral Scientist 19:703-718.

Beltrán, M. y otros (1984), Informe sociológico sobre la juventud española 1960/82. Madrid: Ediciones SM.

Bengtson, V., Furlong, M., y Laufer, R., (1974) "Time, aging and the continuity of social structure: themes and issues in generational analysis", Journal of Social Issues, Vol. 30, n 2 pp1-30 (pp.15-16)

Bettin, G. (1997) "Alcune considerazioni sul mutamento delle generazioni e sul mutamento político", G. Bettin (dir.) Politica e società. Studi in onore di Luciano Cavalli. Padova, CEDAM. 
Bettin, G. (1999) Giovani e democrazia in Europa. Padova:Cedam

Bourdieu P.(1993) "Youth" is just a word" en Bourdieu Sociology in Question. Sage, London.

Bourdieu, (1984). Homo Academicus. Paris: Minuit.

Burgess, W. E. (1968). "Discussion." Pp. 184-205 in Shaw, R. Clifford. The Jack Roller. Chicago and London: The University of Chicago Press. Bolognia.

Buzzi C., Cavalli A., de Lillo A. (1997) Giovani verso il duemila. Il Mulino,

Campbell A. (1960) The American Voter. New York, Wiley.

Carlsson C. (1970) "Age, Cohorts and the Generation of Generations" American Sociological Review vol. 35.

CIS: "Los jóvenes de hoy". Datos de opinión, 19, 1999.

Coser, A. L. (1974). The Pleasure of Sociology. New York: Signet.

Cowgill D. (1974) "The aging of populations and societies" Annals of the American Academy of Political and Social Science. September, vol. 415. $26 n^{\circ} 4$

Crittenden J. (1962) “Aging and party affiliation” Public Opinion Quarterly vol

Cutler N. (1975) "Toward a Generational Conception of Political Socialization" en Schwartz D. ed. New directions in Political Socialization. Free Press, New York.

Cutler N. y Bengtson V. (1974) "Age and Political Alienation: Maturation, Generation and Period Effects" Annals of the American Academy of political and Social Science. Vol. 415.

Cutler S. y Kaufman R. (1975) "Cohort Changes in Political Attitudes: Tolerance of Ideological Nonconformity" Public Opinion Quarterly vol. 39 n¹ .

Dawson R. y otros. (1977) Political Socialization. Little \& Brown. Boston.

Douglas, J. W. B. (1976) "The use and abuse of national cohorts", en M. Shipman (ed.) The Organization and Impact of Social Research. London: Routledge \& Kegan Paul

Edmondson R. (1984). Rhetoric in sociology. Macmillan Press. London. 
Elder, G. (1975) "Age differentiation and the life course", Annual Review of Sociology, Vol. 1. pp.165-90 (p.173)

Fogarty, M. (1973) Forty to Sixty: how we waste the middle age. London: CSSP

Foner, A. (1980) "The sociology of age stratification: a review of some recent publications", Contemporary Sociology, Vol. 9, no.1, pp.771-9

Glenn (1977) Cohort Analysis. Sage.

Glenn N. (1974) "Aging and Conservatism" Annals of the American Academy of Political and Social Science. Vol. 415.

Glenn N. y Hefner T. (1972) "Further Evidence on Aging and Party Identification”. Public Opinion Quarterly, 36.

Glick, C.E. (1955). "Social types in Race Relations." Pp. 194-200 in Race Relations in World Perspective, edited by A.W Lind. Oxford: University Press.

Goffman, E. (1959). The Presentation of Self in Everyday Life. New-York: Doubleday.

Greenstein, F. (1965), Children and politics, New Haven, Yale University Press. Hyman, H. (1959), Political Socialization, Nueva York, Free Press.

Hout M. y Knoke D. (1975) “Change in Voting Turnout, 1952-1972” Public Opinion Quarterly vol. 39 no1

Iglesias de Ussel, J. (1988) "Socialización y control social", en S. Del Campo (ed.), Tratado de Sociología, vol. I. Madrid, Taurus.

Inglehart, R., (1991), El cambio cultural en las sociedades industriales avanzadas, CIS. Madrid.

Jennings, M.K. y Niemi, R. (1981), Generations and Politics, New Jersey, Princeton University Press.

Kaufman R. Y Spilerman S. (1982) "The age structures of occupations and jobs", American Journal of Sociology, Vol. 84, n 4, pp827-51.

Klapp, E. O. (1949). "The Fool as a Social Type." American Journal of Sociology 55: 157-162.

Klapp, E. O. (1958). "Social Types: Process and Structure." American Sociological Review 23: 674-678.

Knoke D. y Hout M. (1974) "Social and Demographic Factors in American Political Party Affiliayions (1952-72)" American Sociological Review, vol 39. 
Linton, R. (1942) “Age and sex categories”, American Sociological Review, Vol. 7, pp.589-603

López Pintor, R. (1982) La opinión pública española del franquismo a la democracia. CIS. Madrid.

Manheim, K. (1952). "The Problem of Generation." Pp. 276-320 in Essays on the Sociology of Knowledge. New York: Oxford University Press.

Martín Serrano, M. (1996) Informe de la juventud en España. Instituto de la Juventud. Madrid.

Navarro, M. y Mateo, M. J. (1993), Informe juventud en España, Madrid, Ministerio de Asuntos Sociales, Instituto de la Juventud.

Neugarten, B., Moore, J. y Lowe, J. (1965) "Age norms, age constraints and adult socialisation", American Journal of Sociology, Vol. 70, pp.710-17 en R.N.

Nissel, M. (1982), "Families and social change since the second world war",

Oppenheim K. y otros. "Some Methodological Issues in Cohort Analysis of Archival Data" American Sociological Review, vol. 38

Orizo, A. (1996) Sistemas de valores en la España de los 90. CIS. Madrid.

Park, B. (1986). "A Method for Studying the Development of Impressions of Real People." Journal of Personality and Social Psychology 51: 907-917.

Park, E. R. (1928). "Human Migration and the Marginal Man." American Journal of Sociology 33: 881-893.

Percheron, A. (1993), La socialisation politique, París, Aarmand Colin.

Perelman, C. (1979) The new rhetoric and the humanities. Dordrecht.

Rapoport, M.P. Fogarty y R. Rapoport (eds.), Families in Britain. London: Routledge \& Kegan Paul

Reading, H. F. (1977). Dictionary of the Social Sciences. London: Routledge and Kegan Paul.

Recchi E. (1997) Giovani politici. CEDAM. Padova.

Rex, J. (1977). "Scientific Laws and Ideal Types: The Sociological Methodology of Max Weber." Canadian Journal of Sociology 2: 151-166. 
Riley, M. (1973) "Aging and cohort succession: interpretations and misinterpretations”, Public Opinion Quarterly, Vol. 37, n 1, pp. 35-49

Riley, M. , Johnson, M. y Foner, A. (1972), Aging and Society, Vol. 3: A Sociology of Age Stratification. New York: Russell Sage

Ryder N. (1965) "The Cohort as a Concept in the Study of Social Change" American Sociological Review, vol 30.

Strong, M. S. (1943). "Social Types in a Minority Group: Formulation of a Method." American Journal of Sociology XLVIII: 563-573.

Strong, M. S. (1946). "Negro-White Relations as Reflected in Social Types." American Journal of Sociology LII: 23-30.

Taub, E. D. and Leger G. R. 1984. "Argot and the Creation of Social Types in a Young Gay Community." Human Relations 37: 181-189.

White Riley M. (1973) "Aging and cohort succession: interpretations and misinterpretations” Public Opinion Quarterly. Vol. 37 n 1

Zárraga, J.L., (1985), Informe juventud en España. La inserción de los jóvenes en la sociedad. Madrid, Instituto de la Juventud. 